\title{
La comunicación de los Objetivos de Desarrollo Sostenible en las organizaciones de la Economía Social
}

\section{José María Herranz de la Casa, Sara García Caballero}

RESUMEN: La agenda 2030 y los Objetivos de Desarrollo Sostenible se han convertido en la referencia que marca la acción de los gobiernos, las naciones, las empresas, las ciudades y los ciudadanos. Cinco años después de su lanzamiento, los datos sobre el cumplimiento de las metas para el año 2030 encuentran áreas críticas y tendencias favorables, lo que hace necesario acelerar el proceso. Las organizaciones de la economía social tienen una oportunidad para posicionarse preferentemente por su filosofía y valores que abarcan la sostenibilidad, la responsabilidad social y la persona. En este artículo se analiza el modelo de comunicación que están desarrollando las principales organizaciones de la economía social en España. Un modelo de comunicación de tres niveles -declarativo, informativo y participativo- que supone un mayor o menor compromiso en la implantación de los ODS dentro de la organización con sus miembros y fuera de ella para visibilizarlos. Se han analizado distintas iniciativas donde la participación de los miembros de organizaciones ha generado reconocimiento en este ámbito. La comunicación no es solo un proceso informativo, sino que supone diálogo y puntos de encuentro para sensibilizar, concienciar y ejercer influencia. Las organizaciones de la economía social no han destacado especialmente por la gestión de la comunicación, pero potenciar la implantación de los ODS puede ser una apuesta firme por diferenciarse en los próximos 10 años.

PALABRAS CLAVE: Comunicación, reporte, sensibilización, cooperativas, sostenibilidad, responsabilidad social, ODS.

CLAVES ECONLIT: L310, L820, M14.

Cómo citar este artículo/How to cite this article: HERRANZ, J.Ma . \& GARCÍA, S. (2021): "La comunicación de los Objetivos de Desarrollo Sostenible en las organizaciones de la Economía Social", CIRIEC-España, Revista de Economía Pública, Social y Cooperativa, 101, 165-191. DOI: 10.7203/CIRIEC-E.101.18393.

Correspondencia: José María Herranz de la Casa, Facultad de Comunicación, Universidad de Castilla-La Mancha, Josemaria.Herranz@uclm.es, ORCID: 0000-0002-3667-2664; Sara García Caballero, Facultad de Comunicación, Universidad de Castilla-La Mancha, Sara.Garcia3@alu.uclm.es, ORCID: 0000-0001-90232937. 


\section{Expanded abstract}

\section{Communication of Sustainable Development Goals in Social Economy organizations}

The 2030 Agenda and the Sustainable development Goals (SDG) are a universal commitment to mobilize resources, a challenge for each State to meet its own goals, a new strategy that must rule world development programs, an action plan in favor of people and the planet. They have become a new moral commitment and the map that sets the course of governments, cities, companies, organizations, and citizens in the next ten years.

Five years after their launch, the perspectives for meeting these goals by 2030 are uneven. There are critical areas and favorable trends in the international sphere (United Nations, 2019; Sachs et al., 2020); in the Spanish landscape, data is not much different, and there is a need to accelerate their implementation (Castiñeira, 2019; Ruiz \& García, 2019; Sánchez et al., 2020). In this context, it is relevant to have indicators to assess and measure the degree of progress of the Sustainable Development Goals. It is also necessary to communicate and make them visible to transform the SDG into an option that allows citizens to choose organizations that offer more sustainable products and services (Silos et al., 2018). Communication is much more than an informative process; it is a management process of the relations between people. When speaking about communication as a space for dialogue and participation, it is necessary to address elements such as identity, perception, and the very action of communication for proper analysis. This article contextualizes current commitment and identification with SDG of companies and organizations; it goes deep into the perceptions and knowledge of citizens of these goals and focuses on SDG communication for social economy organizations.

This research's main objective is to review the contributions of social economy organizations to SDG communication. It departs from a perspective of its development, sensitization, and the raising of awareness towards them. To reach this objective, we start with two hypotheses: The first one, social economy organizations' communication of the SDG is scarce; the second one, SDG communication, responds to a declarative model versus a participative model. The methodology used was a documentary and content analysis of social economy organizations' communication of the SDG in Spain. To accomplish this analysis, we examined different representative families and entities of this sector. We tracked web sites, social media sites, studies, and reports from various organizations. We paid particular attention to organizations with greater economic relevance and representation in Spain, according to CEPES (Confederación Empresarial Española de la Economía Social -Spanish Enterprise Confederation for Social Economy). In total, we analyzed social media publications, web sites, and news spaces of close to a hundred companies between January 2018 and mid-September 2020.

Additionally, we analyzed activities and published news to examine how SDG and the 2030 agenda were communicated, according to the three proposed tiers in the communication mod- 
el. The three levels of the communication model are: declarative, where organizations limits to commit in writing or participate in SDG related events without thoroughly implementing the development of these goals within the organization's policies and defined strategies; informative, where the organization considers developing some of the SDG, inform and offer basic training to its employees and may even produce a report of the achievements in a registry format, and monitors and informs about its progress; and participative, were the members of the organization get involved in the implementation and become the leading prescribers and ambassadors of SDG, and also, the organization participates actively in the diffusion of SDG, with full integration within their communication channels. Finally, the article shows four social economy organization cases representing an excellent example of the communication model's participation level. Regarding the results obtained, the majority of the hundred organizations analyzed have a web presence. However, their presence in the principal social media networks, such as Twitter, Facebook, YouTube, and LinkedIn, is minimal and uneven.

Similarly, only a few of the enterprises include SDG references or news on their web sites. The only ones that stand out with information on their websites are the cooperative organizations COBADU, COPISO, CAJAMAR, Amiab Group, and Espriú Foundation. Out of 28 organizations associated with CEPES, eleven fluctuate between the communication model's informative and participative tiers. The ten organizations are the very Spanish Enterprise Confederation for Social Economy (CEPES), CEPES Aragon, Catalunya Cooperative Confederation (COOPCAT), Comunitat Valenciana Cooperative Confederation (CONCOVAL), The Spanish Confederation of Consumer and User Cooperatives (HISPACOOP), Agro-Alimentary Cooperatives of Spain, Mondragon, Coviran, Federation of Enterprise Associations of Inclusion Enterprises (FAEDEI), Espriú Foundation and ONCE.

The article also analyzes four cases that show the ways a participative communication model may develop. The first case involves Gavina School in Picanya (Valencia), who developed a themed project on human rights and sustainable development goals with students aged 12 to 16 (1ํㅡㄴ to $4^{\circ} \mathrm{ESO}$ ). Teachers, students, and parents were involved in the initiation and development of the educational project linked to the SDG; the second case also coincides with an academic project from the Sorolla Educational Group. It aimed at raising awareness and spreading the SDG. They produced an environmental project with the educative community's participation (students, teachers, families, and service personnel). Various external stakeholders (companies and public offices) also participated; the third case involves an agricultural cooperative, Oleocampo, Cooperative Society of Andalucia. Oleocampo is an example of alignment with the 8th SDG creating quality job opportunities, commitment to biomass and edible oil tourism; and the fourth case, where Fruits de Ponent Cooperative includes all 17 SDG in their daily activities and produces sustainability reports.

Web pages, social media, and sustainability reports are the main windows of communication of any organization with their context. The analysis's practical conclusions do not show a decisive wager for SDG from Spanish Social Economic organizations. Very few of the organizations leading their sectors have a well-defined communication strategy for SDG. The cases that include references of the SDG have a communication model in the declarative tier, where the 
commitment barely extends to the organization's members or towards society. Nevertheless, this does not mean that there is no concern or they are not working, but they are not communicating.

Social Economy Organizations have in their origins SDG specific values, where people, context, and cooperation are fundamental. These values represent an opportunity we can not miss. It is necessary to continue communicating the SDG, communication must be action, and with a time frame of 10 years ahead, there is still much work citizens can do.

Social economy organizations are better positioned to influence sustainability and SDG since they have good identity values to be the transmission belt of these goals. This research also shows the opportunity these organizations have to take the lead. It is a landscape of slow processes, where some goals lay far ahead. Social economy organizations have a chance to communicate their philosophy, values, and alignment with the SDG and the 2030 agenda.

Keywords: Communication, report, sensitization, cooperatives, sustainability, social responsibility, SDG. 


\section{Introducción}

La Agenda 2030 y los ODS se han convertido en el nuevo compromiso moral y en el mapa que marca el rumbo de los gobiernos, las ciudades, las empresas, las organizaciones y los ciudadanos en los próximos 10 años. Todo lo que se desvíe de esas metas, de este lenguaje universal y se salga de los indicadores para medirlo, estará desafiando el devenir global.

Cinco años después de su lanzamiento, las perspectivas de cumplimiento para el año 2030 son dispares en el ámbito internacional, donde se habla de áreas críticas y tendencias favorables (Naciones Unidas, 2019; Sachs et al., 2020); y en el ámbito español, los datos no cambian demasiado y existe la necesidad de acelerar la implantación (Castiñeira, 2019; Ruiz \& García, 2019; Sánchez et al., 2020), puesto que las proyecciones realizadas por algunas investigaciones a 10 años no dejan mucho margen para la espera (Boto-Álvarez \& García-Fernández, 2020). No solo es relevante tener indicadores para evaluar y medir el grado de avance en los Objetivos de Desarrollo Sostenible, sino también visibilizarlos y comunicarlos para que se conviertan en una opción de elección de producto y servicios sostenible para los ciudadanos (Silos et al., 2018).

En este contexto, el papel de las organizaciones de la economía social se torna más relevante por su propia naturaleza y por sus valores que entroncan desde hace mucho tiempo con el desarrollo sostenible, la responsabilidad social y el enfoque en la persona. Su filosofía se sostiene sobre el equilibrio de la perspectiva económica y social, unos pilares sobre los que se cimienta la Agenda 2030 y los ODS. Además, las organizaciones de economía social, por su propia naturaleza, tienen una raíz más comunicacional que el resto de las empresas e instituciones. Esto puede afirmarse porque comunicar significa poner en común, compartir y la economía social se fundamenta en principios como colaborar, cooperar con otros, participar, en definitiva, la pura esencia del significado del concepto comunicar.

La comunicación es mucho más que un proceso informativo. La comunicación es un proceso de relación, es un esfuerzo por lograr la empatía con el otro. Supone diálogo, intercambio, puntos de encuentro, espacios de debate, participación y aquí es donde reside la clave para lograr sensibilizar sobre los ODS. Descubrir cómo están comunicando las organizaciones de la economía social y compartiendo los ODS es necesario para ampliar las fórmulas que extienda su avance.

El objetivo principal de este artículo es realizar una revisión de la contribución de las organizaciones de la economía a la comunicación de los Objetivos de Desarrollo Sostenible, entendida desde la perspectiva de cómo se está haciendo el desarrollo, la promoción, la sensibilización y la concienciación sobre ellos. Para ello, en este texto se despliega un marco teórico sobre el conocimiento, percepción y gestión de la comunicación de los Objetivos de Desarrollo Sostenible en los ámbitos nacional e internacional. Y en el segundo epígrafe, se presentan el diseño metodológico y los resultados de la investigación sobre la comunicación de los ODS en las organizaciones de la economía social, basado en los informes y estudios existentes (CEPES, 2019; Mozas Moral, 2019; CIDEC, 2019) y también en la propuesta investigadora donde se analizan cómo están comunicando las organizaciones de la economía social en sus distintas 
familias y sus entidades representativas del sector, a través de las páginas web, redes sociales, memorias y noticias. El texto finaliza con unas conclusiones donde se esboza una propuesta de estrategia comunicativa para los próximos años.

\section{Marco teórico: conceptos para la gestión de la comunicación de los ODS en la economía social}

Para plantear el marco de la gestión de la comunicación de los ODS en las organizaciones de la economía social, es importante desglosarlo a partir de los tres conceptos clave que plantea Sanz de la Tajada (1994: 42-44) cuando analiza la comunicación en las organizaciones. Este autor plantea tres conceptos básicos que son: la identidad, la comunicación y la imagen. Estas tres dimensiones se van a analizar desde la perspectiva general de los ODS y desde la perspectiva particular de la economía social. De esta manera será posible alcanzar una perspectiva del análisis final.

\subsection{La identidad de los ODS y su conexión con la economía social}

La Agenda de Desarrollo Sostenible, conocida como Agenda 2030, fue ratificada el 25 de septiembre de 2015 en Nueva York por los 193 países que formaban la Asamblea General de Naciones Unidas. En ella se plantearon los 17 Objetivos de Desarrollo Sostenible (ODS), con 169 metas, que se enmarcan en cinco áreas: las personas, el planeta, la prosperidad, la paz y la asociación.

Son la continuidad de los Objetivos de Desarrollo del Milenio (ODM), que se acordaron a comienzos del siglo XXI (2000-2015) para estimular los esfuerzos de cooperación internacional en la lucha por la erradicación del hambre, la pobreza extrema o el cambio climático (Alonso y Ayuso, 2017). Los ODM fueron un punto de partida, pero que tras los 15 años que estuvieron vigentes, el balance presentaba logros, progresos, fallos e incumplimientos (UNDP-MDG Report, 2015).

Los ODS son un compromiso universal para movilizar recursos; un reto de cada Estado por cumplir sus propias metas; una nueva estrategia que debe regir los programas de desarrollo mundial; un plan de acción en favor de las personas y el planeta. No obstante, después de cinco años, los datos de los informes internacionales (Sachs et al., 2020) y nacionales no muestran avances significativos. La pregunta que se plantea aquí es de qué manera los ODS están especialmente vinculados con las organizaciones de la economía social. 
En este año 2020 se cumplen 25 años de la declaración sobre la identidad cooperativa de la Alianza Cooperativa Internacional (ACI), donde se identificaron los valores compartidos del movimiento cooperativo y se añadió el 7ํ principio: Compromiso con la comunidad. Un principio que se alinea con los conceptos de sostenibilidad, responsabilidad social y que llega hasta los ODS. Como señalan Chaves Ávila y Monzón Campos (2018), las organizaciones de la economía social se sitúan en nociones ligadas a la reorientación de los objetivos y modo de funcionamiento de las economías como son la responsabilidad social y la economía del bien común. Son estos conceptos de valor social y económico de estas organizaciones los que reafirman su función como un elemento identitario que se recoge en la Carta de principios de la Economía Social de Social Economy Europe, donde no solo prima en estas organizaciones el interés de los miembros o usuarios, sino también el interés general y los principios de desarrollo sostenible, solidaridad y de responsabilidad.

Las organizaciones de la economía social desde su origen más remoto que es el de las cooperativas, siempre han estado vinculadas con todo ello. La siguiente tabla muestra la vinculación entre los valores cooperativos y los ODS, según Alarcón Conde y Álvarez Rodríguez (2020:74).

\section{Tabla 1. Relación entre los principios cooperativos y los Objetivos de Desarrollo Sostenible}

\begin{tabular}{l|c} 
Principios Cooperativos & ODS \\
\hline Adhesión voluntaria y abierta & 16 \\
\hline Gestión democrática por parte de los asociados & $16,2,9$ y 10 \\
\hline Participación económica de los socios & 16 \\
\hline Autonomía e independencia & $4,8,12,13,14$ y 15 \\
\hline Educación, formación e información & 4 y 17 \\
\hline Cooperación entre cooperativas & $1,2,3,4,5,6,8$ y 10 \\
\hline Compromiso con la comunidad &
\end{tabular}

Fuente: Elaboración propia a partir de Alarcón Conde y Álvarez Rodríguez (2020: 74).

Asimismo, hay otras investigaciones que han buscado la conexión entre los valores de la economía social, de las cooperativas o las Sociedades Agrarias de Transformación (Mendoza Jiménez et al., 2020) con los ODS. La investigación de Mozas Moral (2019:29) ve una relación estrecha entre los ODS 1, 2, 3, 4, 5, 6, 8, 10 y 17 y los valores que defiende la economía social y profundiza en la vinculación del cooperativismo oleícola con doce de los diecisiete ODS (Mozas Moral, 2019: 51).

No obstante, como se analizará en los siguientes apartados, la comunicación de todos estos valores y principios no ha sido su punto fuerte. Y si bien, dejaron pasar la oportunidad de 
posicionarse como organizaciones cuya marca de identidad era la responsabilidad social, en la actualidad, según se analizará más adelante, también están dejando pasar la oportunidad de posicionarse como organizaciones cuya filosofía gira entorno a los ODS. Solo sirva como ejemplo, la corporación Mondragón, símbolo y referente cooperativo internacional, que en su informe anual de 2019 dice:

Finalmente, en relación con la Responsabilidad Social de las cooperativas y la preocupación por la correcta conservación de nuestro entorno, en las cooperativas contamos con 75 certificaciones IS014001 de gestión medioambiental que suponen más del 75\% de las ventas totales de las cooperativas industriales. Además, las normas relacionadas con la responsabilidad social, como la SR10, ya cuentan con algunas certificaciones en las cooperativas. Asimismo, las 20 cooperativas más grandes han realizado por segunda vez el "Estado del Informe No Financiero". No cabe duda de que en los próximos años se seguirấ avanzando en una mejor respuesta a los Objetivos de Desarrollo Sostenible (ODS).

Este texto anticipa el contexto y es un reflejo de una de las hipótesis planteadas en esta investigación y es que los ODS en las organizaciones de la economía social en España no son una piedra angular de la identidad de estas organizaciones, sino que aparece como un apunte que hay que incluir. En los últimos años, los ODS han empezado a desplazar y absorber a los conceptos de sostenibilidad y responsabilidad social empresarial. Según, el último informe del Pacto Mundial de Naciones Unidas (DNV GL, 2020) sólo 46 \% de las empresas tienen dentro del propósito del negocio los ODS, el $37 \%$ han diseñado modelos de negocio que contribuyen a su logro y el $61 \%$ de las empresas proporciona productos y servicios que contribuyen a los ODS, frente al 48\% en 2019. En España, según el informe de Castiñeira (2020: 9), menos de la mitad de las 137 empresas analizadas (47\%) incluyen los ODS en sus memorias anuales de 2018. Entre las empresas que reportan e incorporan los ODS, la mayoría les otorgan un valor estratégico, alineándolos con su estrategia de negocio. Además, según el informe del Pacto Mundial Red Española (Sánchez et al., 2020), un 86\% de las empresas del Ibex 35 incluyen información sobre ODS en sus memorias de sostenibilidad de 2019, pero son pocas las empresas que establecen compromisos específicos para medir y evaluar su contribución, en 2019 únicamente lo hicieron 7 de las 35 empresas.

\subsection{Imagen: La percepción pública de los Objetivos de Desarrollo Sostenible}

Después de cinco años de implantación de los ODS y de la Agenda 2030, las encuestas e informes elaborados a lo largo de estos años sobre el conocimiento de estos por parte de la ciudadanía, dejan datos preocupantes. En España, el barómetro del Centro de Investigaciones Sociológicas (CIS, 2019) de febrero de 2019 (Estudio nํ․ 3240) preguntaba sobre si había oído hablar de la Agenda 2030, y el 85,8\% respondió que no, que era la primera vez. Solo el 13,1\% 
conocía o había oído hablar de ella. En Alemania, el informe del Instituto Alemán para la Evaluación del Desarrollo, German Institute for Development Evaluation (Gleser y Schneider, 2018: 50) mostraba que más del 50\% de los encuestados no habían oído hablar de los ODS en el verano del 2015 y dos años después, en el 2017, los datos eran idénticos. Junto a estas cifras, solo menos del 10\% había oído hablar de ellos y tenía idea de su contenido, frente al 23\% que había oído hablar de ellos, pero no conocía su contenido. Por el contrario, cuando se pregunta a los europeos a través del Eurobarómetro sobre temas relacionados con los ODS, como el medio ambiente o la pobreza, los datos cambian. En el Eurobarómetro de marzo 2020, el 94\% de los ciudadanos europeos pensaba que la protección del medio ambiente es importante y el 91\% que el cambio climático es un problema serio en la Unión Europea. En el Eurobarómetro de octubre de 2019, a la pregunta sobre si la lucha contra la pobreza en los países en desarrollo es también interés de la UE, un 79\% respondió afirmativamente, frente a un 13\% en desacuerdo y un $8 \%$ que no lo sabía.

La conclusión es que existe desconocimiento sobre los ODS y en el proceso de comunicación no se ha conectado la preocupación de los europeos con los temas que están dentro de la Agenda 2030, que al final están en la misma línea.

No obstante, más allá de Europa, cuál es la percepción de los ODS. El afrobarómetro de noviembre de 2018, que encuesta a ciudadanos de 34 países africanos (45.800 personas), apuntaba que los ODS más relevantes son: ODS 8 -trabajo decente y crecimiento económico- con un amplio margen (57\%); a continuación le sigue el ODS 2 -hambre cero- con un 31\%; el ODS 3 -buena salud y bienestar- con un 27\%; el ODS 16 -paz, justicia e instituciones fuertes- con un 26\%; el ODS 9 -industria, innovación e infraestructura- con un 24\% al igual que el ODS 6 -agua limpia y saneamiento-; el ODS 1 -fin de la pobreza- con un 22\%;el ODS 4 -educación de calidad-con un 21\%; el ODS 7 -energía limpia y asequible- con un $13 \%$ y, finalmente, con menos de un 5\% de prioridad estarían el ODS 11 -comunidades y ciudades sostenibles- (4\%); el ODS 13 -acción por el clima-(3\%); el ODS 10 -reducción de las desigualdades- (2\%) y el ODS 5 -igualdad de género- (1\%). Estamos acostumbrados a visiones eurocentristas, y este orden en los datos puede resultar clave para conocer las percepciones de otros continentes y ayudar a su consecución, ya que los ODS son globales, y.

Otro estudio realizado en Pakistán (Javeed et al., 2020), muestra que el cumplimiento y la implementación de los ODS es tarea del gobierno, ya que es quien tiene la autoridad y los recursos para alcanzarlos. Los encuestados comprenden la relevancia de los ODS, pero no muestran mucho interés en el cumplimiento de estas metas, aunque los resultados varían según la región. En China, otro estudio de Guan et al. (2019), donde se analizan las actitudes de los ciudadanos hacia los ODS en cinco ciudades chinas, destaca que los ciudadanos con una cosmovisión más antropocéntrica apoyan más los ODS que aquellos que tienen una visión más ecocéntrica. Esto significa que los ODS se perciben más como una cuestión de política de desarrollo que como una cuestión de política medioambiental.

También demostraron que el papel que juega el conocimiento sobre los ODS es de vital importancia en el fomento del apoyo público. Existe una correlación significativa entre el conocimiento y el apoyo. 
En la última encuesta global de abril 2020, que recoge el informe de la Red de Soluciones para un Desarrollo Sostenible (Sustainable Development Solutions Network / SDSN) (Sachs et al., 2020: 53) el 52\% de los encuestados responden que estamos moderadamente en camino y se podrían logar la mitad de los ODS para 2030, mientras que las principales barreras para alcanzarlos en sus países, según un $67 \%$ es la falta de liderazgo político y para un $53 \%$ la falta de sensibilización de la población y de los políticos.

Todos estos datos muestran que el conocimiento y percepción de los ODS en el mundo es bajo, no se relaciona con los temas que preocupan a los ciudadanos y cambian en función del continente; y además se pone el énfasis en que la responsabilidad de los políticos es prioritaria para que los ciudadanos puedan apoyarlos. También, todos estos informes ponen de manifiesto los ineficientes esfuerzos de comunicación y sensibilización, realizados en muchos países para conocerlos hasta ahora.

\subsection{La gestión de la comunicación de la agenda 2030 en las organizaciones y empresas}

Es muy difícil gestionar la comunicación de la Agenda 2030 y los ODS si no se da una condición esencial: que la comunicación sea relevante para una organización. Este es el primer punto que se abordará aquí teniendo en cuenta la perspectiva de las organizaciones de la economía social. En el segundo, se ofrece la fundamentación de los modelos de comunicación de los ODS.

\subsubsection{La relevancia de la comunicación en las organizaciones de la economía social}

Una de las principales dificultades que siempre ha existido en las organizaciones de la economía social es la amalgama de entidades con sus singularidades que componen este sector. Un hecho que ha impedido, en muchas ocasiones, dotarla de un mensaje unificado para que sean percibidas. Esta dificultad no es solo propia de España, como señalaba hace años Davant (2003:411):

Pese a la diversidad de las estructuras y de las acciones que ha llevado a cabo la economía social tanto a nivel francés como europeo, no ha ganado suficiente transparencia y coordinación. Entre todas estas iniciativas, no existe un hilo conductor lo suficientemente sólido, una línea política clara y coherente. Hemos hecho rimar a menudo diversidad y pluralidad con cacofonía y dispersión. Éstos son, hemos de reconocerlo, los males que ha sufrido y sufre todavía la economía social.

Esta situación también se ha visto amplificada por otra dificultad, que es la escasa y deficiente relevancia de la comunicación en las organizaciones de la economía social en España. Algunos estudios como los de Herranz de la Casa (2007) y Herranz de la Casa et al. (2018) 
muestran que estas organizaciones siguen siendo poco conocidas, a pesar de la relevancia económica en algunos sectores o regiones; en muchos casos su identidad se reduce al cooperativismo, y dentro de este sector al ámbito agrario y rural; llegan a asociarse en ocasiones con organizaciones caritativas u $0 \mathrm{NG}$, debido a que algunas cooperativas de iniciativa social, empresas de inserción o centros especiales de empleo, son promovidos por asociaciones o fundaciones. El trabajo de Martínez-Carrasco Pleite y Eid (2017) en la región de Murcia demuestra que hay regiones como la murciana donde hay un alto nivel de confianza, notoriedad y conocimiento de las cooperativas, pero también manifiesta la confusión entre el sector no lucrativo de las ONGs y las cooperativas. Y en este mismo sentido, la labor de organizaciones como CEPES en el ámbito nacional ha permitido proyectar y unificar un mensaje del sector a lo largo de los últimos años que la ha convertido en un interlocutor a nivel político, social y económico.

También, estos estudios aseguran que en muchas ocasiones los medios magnifican las crisis y fracasos y hay un predominio de visiones negativas e ideas caducas del pasado, fruto de experiencias fracasadas (algunas cooperativas de viviendas). Y todo ello, a pesar de que la economía social ha demostrado su liderazgo, crecimiento, reconocimiento público, proyección internacional, competitividad e innovación.

Todas estas afirmaciones, se consolidan con la suma de otro factor determinante en la comunicación del siglo XXI, la digitalización. Y en este sentido, los últimos estudios muestran muchas similitudes con los datos anteriores. Las investigaciones de Bernal Jurado et al. (2019) demuestran un importante déficit en la calidad de los sitios web de las empresas españolas de producción ecológica. Jorge et al. (2019) ratifican que el 55,46\% de las cooperativas agroalimentarias tienen web corporativa, lo que supone un retraso del sector cooperativo frente al resto de empresas; y los datos de Araujo Batlle et al. (2020) también muestran el bajo número de cooperativas con página web en Cataluña. En cuanto a las redes sociales, los datos de Cristóbal Fransi et al. (2017) sobre las cooperativas oleícolas de Cataluña arrojan resultados como los siguientes: las cooperativas apenas utilizan la web para establecer diálogo con los usuarios y utilizan las vías tradicionales del teléfono y el correo electrónico. Y en cuanto a la interactividad a través de la web 2.0, los resultados demuestran que más del $40 \%$ de los sitios webs analizados disponen de enlaces a la cuenta de microblogging, Twitter y a otras redes sociales como Facebook o LinkedIn.

Estos datos refuerzan el argumento de que la comunicación digital en las organizaciones de la economía social no está desarrollada al mismo nivel que en otras empresas. Esta situación le hace perder competitividad y relevancia, a pesar de su implantación y liderazgo en sectores como el agroalimentario.

\subsubsection{Los modelos de comunicación sobre los ODS}

El concepto de comunicación viene del latín communicare que significa poner en común, compartir o hacer partícipe, y también tiene una acepción con el significado de informar. Desde el punto de vista de la comunicación en una organización, como señala Weil (1992: 192), la 
comunicación en la empresa es más que una técnica, es una filosofía, la expresión de una visión que tiene que ponerse en marcha de forma centralizada. Y en la comunicación es fundamental analizar el grado de coherencia entre lo que somos (identidad), decimos/hacemos (comunicación), lo que perciben (imagen), conceptos que plantea como base Sanz de la Tajada (1994: 42-44).

Por esa razón, el modelo que se plantea aquí a la hora de comunicar los ODS, sigue esta coherencia y también se apoya en las aportaciones de dos autores. Por un lado, Erro Sala (2002: 27) que esboza tres modelos de comunicación desde la perspectiva de la "educomunicación" que ponen el énfasis en los contenidos (comunicación y educación vertical); en los resultados (comunicación y educación persuasiva); o en el proceso (comunicación y educación dialógica); Y por otro lado, desde la aportación de Costa (1995: 293) que plantea la gestión de la comunicación como un sistema nervioso central de las organizaciones y un flujo vital que la recorre, y además como todo el conjunto de relaciones interpersonales de una organización con su entorno, que también son formas de comunicación, es decir, de influencia. A partir de estos planteamientos se puede hablar de tres niveles en el modelo de comunicación:

1. Declarativo. En este nivel la organización se limita a comprometerse por escrito o a participar en eventos relacionados con los ODS, sin que el desarrollo de estos se incorpore plenamente en la política y la estrategia definida de la organización.

2. Informativo. En este nivel la organización se plantea desarrollar alguno de los ODS, dar difusión y formación básica a los empleados. Incluso puede llegar a realizar un informe con los logros en formato de memoria y monitoriza e informa sobre sus avances.

3. Participativo. En este nivel se involucra a los miembros de las organizaciones para que se impliquen en la implantación. Estos se convierten en embajadores de los ODS y los principales prescriptores. De la misma manera, la organización participa activamente en la difusión de los ODS y en sus canales de comunicación aparece plenamente integrado.

Aunque el SDG Compass (2015) propone la comunicación como el quinto paso a la hora de seguir el alineamiento de las estrategias empresariales con los ODS, la gestión de la comunicación es fundamental en todo el proceso y en los cuatro pasos anteriores para entender los ODS: entender los ODS, definir prioridades, establecer objetivos e integrarlos en el día a día. Si la comunicación se limita a informar sobre la consecución de las metas, se convertirá en una mera suma en la cuenta de resultados.

Los distintos niveles en el modelo de comunicación suponen pasar de una menor a una mayor implicación en el grado de compromiso de la organización con respecto a la Agenda 2030 y la consecución de los ODS. Y también supone una mayor participación de los miembros de la organización. No hay que olvidar que los ODS no son solo una cuestión de empresas o gobiernos, también es de todos los ciudadanos, y es por ello, que la influencia que se puede hacer en el puesto de trabajo se puede trasladar a la vida familiar y a la vida como ciudadano. 


\section{Hipótesis de trabajo y metodología}

En este texto se plantean dos hipótesis de trabajo con las que se pretende lograr el objetivo principal de esta investigación que es conocer la contribución de las organizaciones de la economía a la comunicación de los Objetivos de Desarrollo Sostenible.

H1. La comunicación de los ODS por parte de las organizaciones de la economía social es escasa.

Esta primera hipótesis determina que la comunicación de los ODS sigue la misma línea de gestión de la comunicación de las organizaciones de economía social. Como se ha reseñado anteriormente, esta tipología de organizaciones no se ha caracterizado por el desarrollo de estrategias de comunicación sólidas a lo largo de su historia. Un hecho que ha contribuido a un desconocimiento de su relevancia en los territorios. En esta misma línea, la importancia de estas organizaciones en el plano económico, social y medioambiental no está siendo objeto de comunicación a la hora de alinearse como organizaciones comprometidas con la Agenda 2030 y los ODS. Esta hipótesis es especialmente clave dado que han pasado cinco años desde la puesta en marcha de los ODS y quedan diez años por delante.

H2. La comunicación de los ODS responde a un modelo de comunicación declarativo e informativo frente a un modelo participativo.

La segunda hipótesis intenta verificar si las acciones de comunicación realizadas por las organizaciones de economía social responden más a un modelo de comunicación declarativo e informativo que participativo. En el punto anterior se ha propuesto estos conceptos que marcan el análisis. Si las organizaciones de economía social solo han adquirido compromisos que tienen relación con su propia identidad, están dejando pasar una oportunidad excepcional para ponerse a la cabeza del cambio social. Sin embargo, si están pasando a la acción y están poniendo el énfasis en el proceso con sus miembros, su capacidad de influencia será decisiva para alcanzar las metas que plantean muchos de los ODS.

En cuanto a la metodología para se ha realizado un análisis documental y de contenido sobre la comunicación de los ODS de la economía social en España. Para ello se han examinado las distintas familias y entidades representativas del sector. Se han rastreado los portales web, las redes sociales y los estudios e informes de las distintas organizaciones que tienen mayor relevancia económica y representatividad en España. Se han analizado las 30 cooperativas con mayor facturación en el año 2018, las 10 empresas con mayor facturación de cada familia, las 9 cofradías, y los 8 grupos empresariales, que aparecen en el documento de CEPES (2018) y UNACC (2019), que suman 97 organizaciones.

Además, se han analizado las actividades y noticias que han generado todas ellas para examinar de qué manera informan y comunican sobre la consecución de los ODS y la Agenda 2030. Y también se han analizado las 28 organizaciones que son miembros socios de CEPES.

Por último, se ha intentado localizar buenas prácticas que representen el nivel participativo del modelo de comunicación que aquí se ha planteado. Asimismo, en el análisis se ha incorporado una breve perspectiva internacional, que permite contextualizar mejor el estado de la cuestión, pero con la pretensión de ser un punto de enfoque más que un análisis exhaustivo. 


\section{Resultados}

\subsection{La comunicación de los ODS en las organizaciones de la economía social}

En este punto se detallan los principales marcos de referencia internacional y nacional en cuanto a la comunicación y difusión de los ODS en el ámbito de la economía social. A continuación, aparecen todos los resultados de los análisis realizados en las páginas web de las principales empresas y organizaciones representativas de la economía social en España. El último punto se centra en cuatro casos donde el modelo de comunicación responde al nivel participativo, el que mayor compromiso supone para una organización con los ODS.

\subsubsection{El marco de referencia internacional y nacional}

Para empezar, se ofrece un pequeño detalle de la comunicación de los ODS en el ámbito internacional, ya que esta marca significativamente los primeros cinco años de implantación de los ODS en los ámbitos nacionales. En primer lugar, la OIT (2014) fue la primera en poner sobre la mesa la relevancia de las cooperativas de cara a la puesta en marcha de los ODS. En segundo lugar, hay que destacar la iniciativa de la Alianza Cooperativa Internacional (ACI) a través de la plataforma Cooperativas hacia 2030 (https://cutt.ly/Cf8CoEZ). Se puede decir que es la primera iniciativa con carácter comunicativo y divulgativo que surge en julio de 2016. El objetivo es que las cooperativas tengan un mayor conocimiento de los ODS y convertirlo en un espacio para recoger los compromisos y las contribuciones de las cooperativas al logro de los ODS. Otro órgano creado por la ACI, y que en estos momentos centra sus esfuerzos en la sensibilización de los ODS es el Comité para la Promoción y el Avance de las Cooperativas (COPAC). A través de una serie de pequeños informes: "Transformando nuestro mundo: una cooperativa 2030", pretende concienciar y mostrar el trabajo desarrollado por las empresas cooperativas en el logro de la Agenda 2030.

Con motivo de la celebración del Día Internacional de las Cooperativas, que se celebra anualmente cada primer sábado del mes de julio, desde hace varios años, cada año se destaca un tema en relación con los ODS. En el año 2020 ha sido el ODS 13 (acción por el clima) \#Coops4ClimateAction; en el año 2019 fue el ODS 8 (trabajo decente y crecimiento económico); y en el 2018, el ODS 12 (producción y consumo responsable).

En el marco de la Agenda 2030 y los ODS, más centrado en el ámbito de la economía social de no mercado, las organizaciones sociales siguen cumpliendo su labor de vigilancia y observación de las acciones realizadas por los gobiernos y las empresas. El SDG Watch Europe (https://cutt.ly/vfR00ty) es una entidad constituida por organizaciones civiles y del tercer sector que se encarga de examinar y monitorizar los informes de la Comisión Europea y las políticas europeas desde la dimensión del desarrollo sostenible; y además aporta propuestas y soluciones que sumen el compromiso de los ciudadanos con los ODS. 
El Grupo de Trabajo Interinstitucional de las Naciones Unidas sobre la Economía Social y Solidaria (UNTFSSE) tiene una plataforma SSE Knowledge Hub (https://cutt.ly/Xf8Cs00) cuyo objetivo principal es aumentar la visibilidad en el ámbito de los resultados de la investigación sobre economía social y solidaria y su contribución a los ODS. Recoge en su web todo un conjunto de documentos de investigación que permiten conocer ejemplos sobre los logros de la economía social y solidaria en la línea del logro de la Agenda 2030.

En el ámbito internacional, el informe del World Cooperative Monitor (https://cutt.ly/vf8B$\mathrm{jZH}$ ) presenta anualmente el impacto económico y social de las mayores cooperativas de todo el mundo y establece una clasificación de las 300 empresas más relevantes. Además, en cada edición analiza la contribución de las empresas a la consecución de los Objetivos de Desarrollo Sostenible (ODS). En el año 2019 se ha centrado en el ODS 8 (trabajo decente y crecimiento económico) y de cara al monitor del 2020 está trabajando en los logros del ODS 13 (acción por el clima).

Por último, hay que señalar la puesta en marcha del Observatorio Estadístico 2020, dentro de la Oficina Regional de Cooperativas de las Américas, que pretende recoger datos que permitan la visibilización y logros de los ODS en las cooperativas.

Todos estos ejemplos estarían en los niveles primero y segundo del modelo de comunicación. En muchos casos son organizaciones que deben ser motivadoras e influenciar en el desarrollo de los ODS en el sector de la economía social.

En el ámbito nacional hay que destacar la labor de CEPES que siempre ha marcado la pauta comunicativa de la economía social en los últimos años y de la misma manera, lo está haciendo en la comunicación de los ODS. Ha realizado cuatro informes sobre la experiencia de las empresas españolas de la economía social en la cooperación al desarrollo. En el último informe de 2019 ya está explícitamente la vinculación con los ODS. El presidente de CEPES, Juan Antonio Pedreño, afirmaba en este cuarto Informe sobre la contribución de la economía social a los Objetivos de Desarrollo Sostenible que "está preparada para contribuir a hacer realidad la Agenda 2030" (CEPES, 2019). En este informe la percepción del tejido asociativo de CEPES de los ODS a los que contribuye sus actividades y proyectos es amplia y está en especial vinculado con los ODS relacionados con el empleo, la reducción de las desigualdades económicas y sociales, el desarrollo local, la igualdad de género, el acceso a una educación de calidad para todas las personas o la inserción en el mercado laboral de colectivos en exclusión. De la misma manera, la Estrategia de Economía Social 2017-2020, publicada en marzo de 2018, marcó las directrices básicas para la promoción de la participación del sector de la economía social en el diseño e implantación de los ODS.

En cuanto al ámbito de la economía de no mercado, entidades del tercer sector, autores como Salamon y Haddock (2015) fueron los primeros autores en destacar su importancia para el logro de cada uno de los Objetivos de Desarrollo Sostenible, conscientes de su contribución en muchas de las áreas. Está en su ADN el reportar, comunicar continuamente y sensibilizar a la población en torno al Desarrollo Sostenible (Carricondo et al., 2019). En España, las organizaciones del tercer sector han incorporado progresivamente en su trabajo diario la agenda 2030 y los ODS. El Tercer Plan Estratégico del Tercer Sector Social (III PETSAS 2017-2021) 
estructura sus actuaciones en tres planos que entroncan con la Agenda 2030: las personas, la relación con los agentes clave y la organización interna (Red Española, 2019: 17). De hecho, la Plataforma de ONG de Acción social, junto a un conjunto de distintas organizaciones sociales, han participado en el último informe de Progreso 2020 sobre los ODS con la contribución de estos actores en la consecución de los Objetivos de Desarrollo Sostenible (Gobierno de España, 2020).

El Comité Español de Representantes de Personas con Discapacidad (CERMI) es una de las organizaciones que ya ha realizado una primera aproximación de sus miembros y su sector al marco de los ODS (Pérez Bueno, 2019). OXFAM/Intermón, UNICEF Comité Español y WWF España también presentaron en marzo de 2019 el documento "Agenda 2030, la urgencia de una visión compartida: un país responsable con la naturaleza y las personas". En él, estas tres organizaciones muestran algunos principios y prioridades para desarrollar una estrategia de Desarrollo Sostenible para España, así como una batería de más de 100 indicadores para España.

La Plataforma del Tercer sector, compuesta por más de 30.000 entidades sociales, presentó en abril de 2020 al Ministerio de Asuntos Económicos y Transformación Digital del Gobierno de España un documento donde se recogen propuestas al Programa Nacional de Reformas 2020 con el objetivo de defender el Estado del Bienestar. Este documento ha sido elaborado por la Red Española de Lucha contra la Pobreza en España (EAPN-España) para promover un desarrollo social y sostenible más equitativo en línea con el Pilar Europeo de Derechos Sociales (PEDS) y los ODS de la Agenda 2030, con los que España se ha comprometido. Además, la Plataforma del Tercer Sector (2020) ha publicado, junto con la Plataforma del Voluntariado de España y Fundación La Liga, una guía sobre voluntariado y deporte para dar a conocer e impulsar los ODS en las organizaciones y voluntarios del tercer sector.

Como puede verse, en los últimos años, las organizaciones del tercer sector se están poniendo al día para proponer, sensibilizar y comunicar la Agenda 2030 y los ODS. Muchas de estas aportaciones están recogidas en el último informe de progreso del gobierno de España (2020).

\subsubsection{Análisis de la comunicación de los ODS de las empresas y organizaciones representativas de la economía social en España}

En este apartado aparecen los datos analizados de las organizaciones más relevantes de la economía social a partir de los informes de CEPES (2018) y UNACC (2019). Se han analizado las redes sociales, las webs y los espacios de noticias de las 97 empresas más relevantes hasta el 15 de septiembre de 2020.

Los resultados que muestra la tabla 2 corroboran que las empresas de la economía social más relevantes en su mayoría tienen web corporativa, aunque en las redes sociales su participación en mucho menor, salvo el caso de las cajas rurales y de los centros especiales de empleo. En cuanto a las webs que cuentan con un apartado de noticias, las diferencias también son grandes en función de la tipología, y en este punto destacan los centros especiales de empleo y los grupos empresariales. A partir de este dato, se analizaron los espacios de noticias y se buscaron las noticias que hacían referencia a los ODS desde enero de 2018 hasta el 15 de 


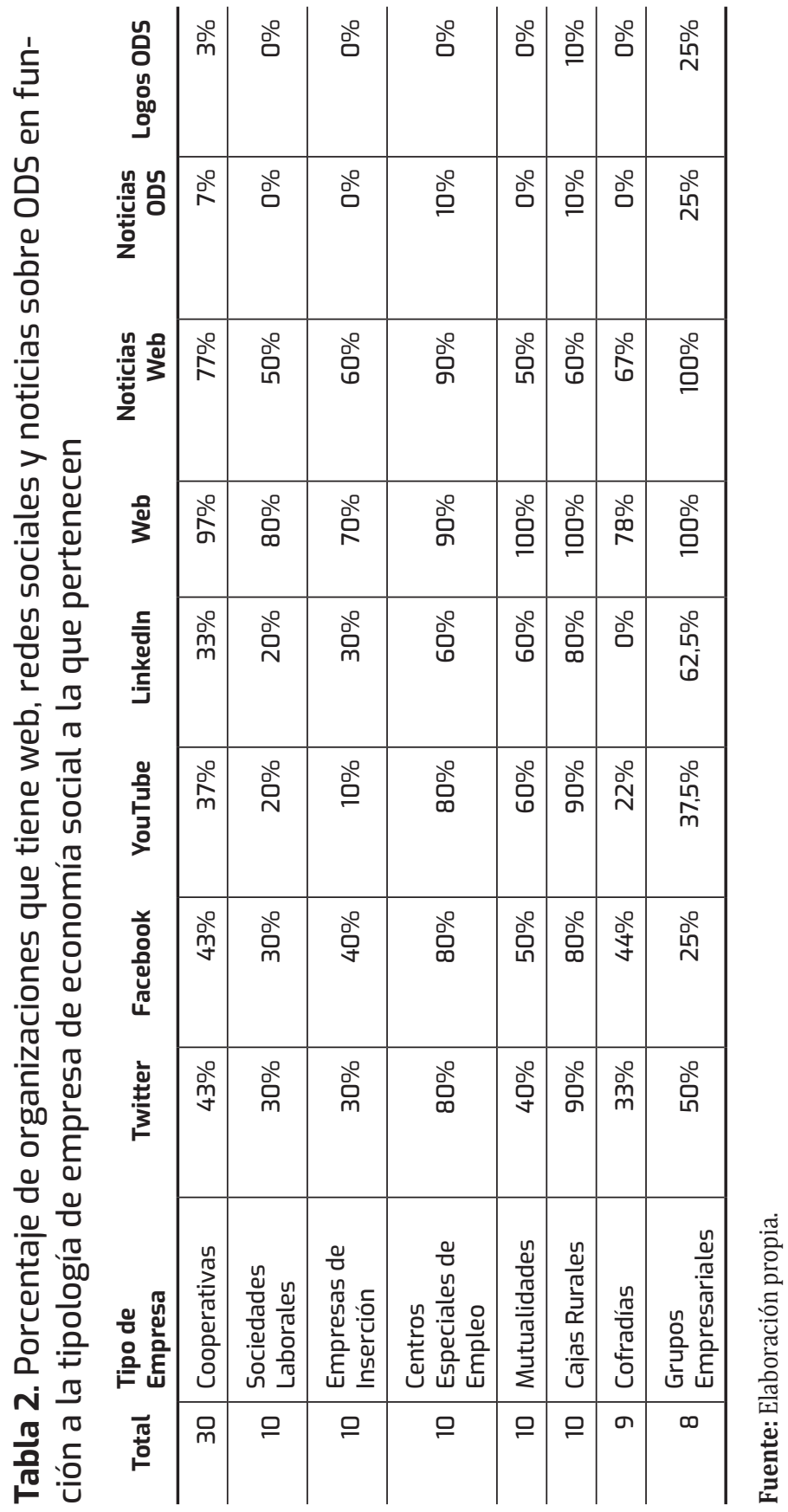


septiembre de 2020. La tabla 2 muestra el porcentaje de empresas de cada familia que tienen noticias sobre los ODS. Son pocas y se desglosan en la tabla 3.

Por último, la última columna de la tabla 2 muestra el porcentaje de organizaciones que utilizan los logotipos de los ODS en sus webs y estas organizaciones son: CAJAMAR, COPISO y la Fundación Espriú.

La tabla 3 analiza las cinco organizaciones que contienen noticias en sus webs sobre los ODS y las temáticas que abarcan: las cooperativas Cobadú (CAJAMAR está duplicada en Cajas Rurales y Grupos empresariales). Como se puede ver en la tabla, las temáticas de las noticias y los ODS a los que corresponden son distintos en función de la actividad empresarial. Los ODS que prevalecen son los de: educación de calidad (4), industria, innovación e infraestructuras (9), producción y consumo responsables (12), acción por el clima (13) y alianzas para lograr los objetivos (17).

\section{Tabla 3. Empresas y organizaciones con noticias sobre ODS y las temáticas que tienen}

\begin{tabular}{|c|c|c|}
\hline Empresas & ODS & Temática noticias \\
\hline S. Coop. Cobadu & $\begin{array}{l}9,12,13,15 \\
\text { y } 17\end{array}$ & $\begin{array}{l}\text { La producción sostenible, la responsabilidad social corporativa, } \\
\text { la sostenibilidad de los sectores ganadero, porcino y rural, el } \\
\text { cooperativismo y la rentabilidad de las infraestructuras rurales } \\
\text { sostenibles. }\end{array}$ \\
\hline $\begin{array}{l}\text { Copiso Soria S. } \\
\text { Coop. }\end{array}$ & $\begin{array}{l}3,7,9,11,12 \\
13 \text { у } 17\end{array}$ & $\begin{array}{l}\text { Desarrollo sostenible, cooperativismo, responsabilidad social } \\
\text { corporativa, apoyos sociales, bienestar y bioseguridad, energías } \\
\text { alternativas, emisión de gases, innovación. }\end{array}$ \\
\hline Grupo Amiab & $\begin{array}{l}4,8,10,12 \\
\text { y } 13\end{array}$ & $\begin{array}{l}\text { Formación, concienciación (campañas y concursos), inclusión y } \\
\text { activación de empleo en discapacitados. }\end{array}$ \\
\hline Cajamar & $\begin{array}{l}4,5,8,9,12 \\
13 \text { y } 17\end{array}$ & $\begin{array}{l}\text { Proyectos de sostenibilidad y economía productiva, } \\
\text { cooperativismo y pactos (Principios de Banca Responsable de } \\
\text { la ONU), igualdad, concienciación medioambiental y del entorno } \\
\text { rural, socialmente responsables con la plantilla, formación, } \\
\text { inversión en innovación. }\end{array}$ \\
\hline Fundación Espriú & 3,4 y 17 & $\begin{array}{l}\text { Publicación Revista de cooperativas sanitarias y medicina } \\
\text { social, "Comprometidos con los Objetivos de Desarrollo } \\
\text { Sostenible"; cooperativismo internacional y alianzas, } \\
\text { sostenibilidad sanitaria y educación para la sostenibilidad de } \\
\text { las cooperativas. }\end{array}$ \\
\hline
\end{tabular}

Fuente: Elaboración propia.

Mientras que las cooperativas como COBADU y COPISO centran sus noticias en la sostenibilidad y las soluciones energética, organizaciones como el Grupo Amiab (centro especial de empleo) centra su información en las distintas actividades de sensibilización y concienciación que realiza con los ciudadanos. El grupo empresaria CAJAMAR destaca por los compromisos 
con los trabajadores y los clientes y, por último, el otro grupo empresarial, la Fundación Espriú, centra su información especialmente en su actividad matriz que es la sanitaria.

Por último, se realizó un mapeo de 27 de 28 organizaciones con web que son socios de CEPES. Se ha analizado si estas organizaciones, que representan a las distintas familias de la economía social, desarrollan acciones de comunicación y en qué nivel estarían en función del modelo: declarativo (1), informativo (2), participativo (3).

El análisis se centra en comprobar si estas organizaciones cuentan con informes y memorias sobre Objetivos de Desarrollo Sostenible y Agenda 2030, enlaces y recursos relativos a los ODS y Agenda 2030; si usan los logotipos de los objetivos de la ONU; si realizan eventos que versen sobre ODS; si tienen noticias sobre Desarrollo Sostenible, ODS y Agenda 2030; si realizan mediciones internas o externas de los ODS y la contribución que hace la empresa a cada uno de ellos o cualquier tipo de formación en torno a los objetivos y metas a cumplir en 2030.

Los resultados son muy variados, ya que hay 11 organizaciones que se acercan bastante a los niveles 2 y 3 -informativo y participativo-. A continuación, se explica las contribuciones de estas organizaciones para ubicarlas en cada nivel. La primera sería la Confederación Empresarial Española de la Economía Social (CEPES) que cuenta con un informe sobre la contribución de la Economía Social a los ODS, recursos multimedia sobre una jornada de la contribución de la economía social a los ODS, usa el logotipo de los ODS en la página de inicio de la web y también tienen una noticia sobre el reconocimiento que Corresponsables hizo en febrero de 2020 a CEPES. Después, se encuentra CEPES Aragón, que tiene enlaces de interés respecto a los ODS como su observatorio, su blog y los casos de éxito que contribuyen a cumplirlos. También utilizan el logotipo universal de los ODS en su perfil de Facebook y organizaron una Jornada sobre economía social y ODS en septiembre de 2019.

La Confederació de Cooperatives de Catalunya (COOPCAT) también se suma a los ODS y promocionó el hashtag con motivo del Día Internacional de las Cooperativas, al igual que la Confederació de Cooperatives de la Comunitat Valenciana (CONCOVAL), que también se ha sumado a una campaña de apoyo a los ODS, promovida por la Red Española del Pacto Mundial. La Confederación Española de Cooperativas de Consumidores y Usuarios (HISPAC00P), por su parte, creó la campaña "Tú cierras el círculo. Cambia a la economía circular", para ayudar al ODS 12 y "FSC Friday 2019", jornada para ayudar al mismos Objetivo.

Las Cooperativas Agroalimentarias de España forma parte de AgriCOOPDS, una iniciativa de promoción del cooperativismo agrario ante el reto de los ODS (https://cutt.ly/Jf80xrF). Aquí se hace una medición sobre la relación de los ODS y la contribución que hace la agricultura familiar a cada uno de ellos.

En el caso de Mondragón, cuentan con la publicación Tu Lankide, donde se habla de los ODS y donde también hay una sección de noticias en las que tratan varios ODS, como, por ejemplo, noticias sobre informes de la ONU, hablando de los ODS 3, 5, 7 y 17; una noticia sobre la plataforma "Cooperativas hacia 2030", que es una campaña de la alianza que unifica a las cooperativas o una noticia sobre que el patronato de Basque Culinary Center aprueba el Plan Estratégico de Desarrollo Sostenible 2020-2024. 
Covirán también se ha sumado a la campaña de apoyo a los ODS promovida por la Red Española del Pacto Mundial y utiliza el logotipo de todos los ODS en su página web. FAEDEI también utiliza el logotipo en su web y ha desarrollado tres jornadas: DEI2019 - Empresas inclusivas en el marco de la AGENDA 2030 en 2019; la XVII Jornada Estatal de empresas de inserción en el mismo año; y la DEI-2018, XVI Jornada Estatal de empresas de inserción: presente, pasado y futuro.

La Fundación Espriú es colaboradora afiliada de la Alianza Cooperativa Internacional y ha participado en la creación del informe World Cooperative Monitor 2018. Además, cuentan con la Revista Compartir, que en el número 1 de nueva época de marzo de 2020, habla sobre los ODS 3, 4, 5, 8, 10, 12, 13 y 17 (https://cutt.ly/Gf8PGjl) y en la web se encuentra una entrevista a Carlos Zarco en Radio Exterior (RTVE), con motivo del 30ํaversario de la Fundación Espriú, hablando de la Agenda 2030.

Por último, en el grupo de las empresas de los niveles 2 y 3, estaría la ONCE y su Fundación, con un Informe de valor compartido 2019 y un informe del Estado de Información No financiera y Diversidad 2019, donde exponen la contribución a los ODS por parte de diferentes organizaciones. También publican la Revista Perfiles, en cuyo número 356, hablan de la contribución a los ODS por parte de varias empresas como La Caixa. Algunos de los eventos que habrían organizado, en relación a los ODS, son: World Blidness Summit Madrid 2020; side event junto con la Organización de Estados Americanos en su compromiso con la Agenda 2030; promoción del evento Maratón Valencia Trinidad Alfonso EDP o la I Jornada de los European Development Days, con el Grupo Social ONCE al frente.

Entre el nivel 1 y 2 se encontrarían ANDESCOOP, AEDIS, COCETA, FEACEM, CAJAMAR, ya que solo cuentan con noticias puntuales sobre Agenda 2030 y ODS, sin participar en eventos y utilizando los logotipos solo puntualmente. Y en el nivel más bajo, sin llegar incluso al número 1 estarían FOROESGAL, CONCOVI, CEPES Navarra, CNEPS, FECOMA, FNCP, Grupo ATLANTIS, Grupo Clade, REAS, UECOE, UNACOMAR y LABORPAR, ya que no tienen ningún informe o memoria relacionada por ODS, no tienen recursos, noticias ni eventos sobre ODS y tampoco utilizan los logotipos en ningún documento ni en sus webs.

No nos gustaría finalizar este apartado sin señalar la apuesta de algunas organizaciones de la economía social a la hora de visibilizar los ODS. Algunas de ellas lo hacen añadiendo en sus redes sociales el logotipo arcoíris de los ODS en su imagen como: FEVECTA (Twitter/Facebook), IUDESCOOP (Facebook/Twitter) o CEPES Aragón (Twitter/Facebook). Son pequeñas acciones que pueden ayudar a saltar de nivel en el modelo de comunicación.

\subsubsection{Buenas prácticas de comunicación en las organizaciones de la economía social}

En este punto se profundiza en cuatro casos donde se puede hablar de las organizaciones que han alcanzado el tercer nivel participativo del modelo de comunicación. Estos ejemplos demuestran el camino hacia el que podrían dirigirse los esfuerzos de otras organizaciones en los próximos años. 
El primer caso corresponde a la Escuela Gavina en Picanya (Valencia) que desarrolló un proyecto temático sobre los Derechos Humanos y los Objetivos de Desarrollo Sostenible con alumnos de entre 12 y 16 años ( $1^{\circ}$ a $4^{\circ}$ ESO). En este blog (https://cutt.ly/Sf8C5Gg), se pueden encontrar todas las propuestas realizadas por los alumnos en el curso 2019-2020 que incluyen desde videos hasta publicaciones, imágenes y presentaciones. Esta propuesta sigue la línea de la 10ํㅡㄹ Declaración Educativa de la Unión de Cooperativas de Enseñanza de la Comunidad Valenciana (UCEV). Esta declaración presentada en abril de 2019 proclamaba el compromiso de la asociación y de sus asociados con los ODS y la necesidad de incorporarlos, "de una manera más sistemática y critica, en los objetivos educativos y en las prácticas de gestión que el cooperativismo educativo desarrolla con un determinado esfuerzo ético y pedagógico".

Este es un ejemplo muy claro de cómo el modelo de comunicación llega hasta el nivel de participación, y donde se involucra a profesores, alumnos y también padres (https://cutt.ly/ Nf8C8W8) en la puesta en marcha y desarrollo del proyecto educativo, vinculado a los ODS.

El segundo caso también se corresponde con un proyecto educativo del Grupo Sorolla Educación. Como señala Teresa Ramos Navarro (2019), la estrategia del grupo cooperativo se alinea con los ODS bajo dos proyectos compartidos: por un lado, en la concienciación y difusión de los ODS y por otro, a través del proyecto medioambiental. En ambos están involucrados toda la comunidad educativa (alumnado, profesores, familias y personal de servicios) tanto en la elaboración y reparto de materiales sobre los ODS, como en la participación en eventos, publicaciones y toda la difusión en la web y las redes sociales. De la misma manera, cuentan con la colaboración de distintos grupos de interés externos en el desarrollo de estas acciones como el ayuntamiento de Valencia y Elche, la Fundación Cajamar o la ONCE y la Asociación Española contra el Cáncer. Asimismo, cuenta con una memoria de responsabilidad social donde los ODS son el eje vertebrador del trabajo que realizan (https://cutt.ly/ef8C9CF). Este caso sigue la misma línea del modelo de comunicación en el nivel de participación y se ha ido consolidado desde el curso 2017-2018.

El tercer caso se corresponde con una cooperativa agraria: Oleocampo Sociedad Cooperativa Andaluza (https://cutt.ly/Gf8C2oc). Según los datos aportados por Mozas Moral (2019: 98-100), Oleocampo es una cooperativa de primer grado fruto de la fusión de tres almazaras cooperativas con más de cincuenta años de historia y es un ejemplo de alineamiento con el ODS 8 creando empleo de calidad, comprometido con la biomasa y el oleoturismo. De la misma manera, el grupo Interóleo se ha convertido en julio de 2020 en el primer grupo comercializador de aceite de oliva del mundo en tener el sello sostenibilidad concedido por la Fundación Copade. Esta certificación verifica la implantación de los ODS dentro del proyecto "Pymes y ODS". Aunque no es mucha la información sobre el trabajo que desarrolla la cooperativa, el hecho de ser reconocida con una certificación por el trabajo de implementación de los ODS supone un paso muy relevante en la comunicación de estos. En este sentido, también hay que decir que la empresa no destaca por su comunicación externa, lo cual no le resta relevancia al trabajo que desarrollan internamente con sus socios.

El cuarto caso es el de la cooperativa Fruits de Ponent, creada en 1992, que conforman hoy cuatro cooperativas de producción frutícola. Esta empresa siempre ha estado a la vanguardia 
de la sostenibilidad y responsabilidad social, incorporándolos en su actividad empresarial diaria. Desde el año 2012 firmó los 10 compromisos del Pacto Mundial de Naciones Unidas, que son un precedente de los ODS. En 2017 se convierte en "socio signatario" de la Red española para el Pacto Mundial, incorporando los 17 ODS a su actividad diaria. Sus buenas prácticas se pueden ver en las memorias de sostenibilidad y en el plataforma del Pacto Mundial (https:// cutt.ly/Uf8CMAl), trabajando especialmente los ODS 1, 3, 5, 7, 8, 12 y 17.

Además, esta cooperativa participa en AgriCOOPDS, una iniciativa liderada por el Foro Rural Mundial que promueve la Agricultura Familiar y el Desarrollo Rural Sostenible. Esta iniciativa tiene el objetivo general de promover el cooperativismo o asociacionismo agrario, aprovechando la oportunidad que nos ofrecen para ello los Objetivos de Desarrollo Sostenible (ODS). La participación en la iniciativa permite la realización de un informe de autodiagnóstico sobre la contribución de la cooperativa a la consecución de los ODS y recomendaciones para seguir profundizando en su compromiso con el desarrollo sostenible. Y también genera una nota de prensa basada en las respuestas del autoinforme para que pueda ser enviado a los medios de comunicación.

La cooperativa incluye dentro de su web y redes sociales los logotipos de Pacto Mundial y de los ODS, con lo cual están también presentes permanentemente para los que los visitan.

\section{Conclusiones}

La comunicación de la Agenda 2030 y los Objetivos de Desarrollo Sostenible (ODS) tiene buenos ejemplos en gobiernos como el sueco; ciudades como Nueva York, Helsinki, Bristol o Los Ángeles; la Universidad de Bolonia, la Universidad de Auckland, la Universidad de Leeds o la Universidad de Bergen; y un gran número de empresas (Herranz de la casa y García, 2020). En el ámbito de la economía social también hay algunos ejemplos, pero el recorrido todavía que hay que hacer es largo.

El análisis realizado corrobora las dos hipótesis planteadas en el trabajo, la comunicación de los ODS en la mayoría de las organizaciones de la economía social es escaso, al igual que su apuesta por la gestión de la comunicación en general. Muy pocas organizaciones, que lideran en sus sectores, tienen una comunicación de los ODS definida. Además, en los casos en los que existen referencias a los ODS, el modelo de comunicación, en la mayoría, responde a un nivel declarativo, donde el compromiso apenas se extiende hacia los miembros de la organización o hacia la sociedad.

Las páginas web, las redes sociales o los informes son la principal ventana de comunicación de cualquier organización con el entorno. El análisis realizado no muestra una apuesta decidida por los ODS, salvo en contadas ocasiones. Sin embargo, esto no quiere decir que no exista la preocupación o no se esté haciendo. Las organizaciones de la economía social tienen en su génesis los valores propios de los ODS en los que las personas, el entorno y la cooperación son fundamentales. Y esta es una oportunidad que no pueden perder. 
Ya sucedió con la responsabilidad social, donde las empresas de economía social no supieron posicionarse y está volviendo a pasar con los ODS. El resto de las empresas ya están viendo sus oportunidades de negocio en la promoción de estos objetivos, a pesar de que su implantación les pueda resultar más difícil y ajena. Muchas organizaciones de la economía social tienen alineada su actividad con un conjunto de ODS, sin embargo, no lo comunican ni interna ni externamente. Este hecho no contribuye de manera global a la consecución de los ODS para el año 2030 y provoca que estén perdiendo una oportunidad competitiva de cara a mostrar su apuesta por la sostenibilidad y la responsabilidad social. Al final es un camino de todos, pero las organizaciones de la economía social están mejor posicionadas de partida.

En el artículo se han destacado cuatro casos que muestran el camino que puede llevar a un modelo de comunicación participativo, un principio fundamental de la economía social. Es necesario seguir comunicando los ODS, la comunicación debe ser acción y con el marco de diez años por delante sigue habiendo mucho trabajo por hacer entre los ciudadanos.

Las organizaciones de la economía social están en mejor posición de influenciar sobre la sostenibilidad y los ODS en el entorno. Estas tienen los valores identitarios adecuados para ser una correa de transmisión de los Objetivos de Desarrollo Sostenible. El trabajo con los distintos grupos de interés, desde los socios hasta los ciudadanos, pasando por los medios de comunicación, pueden permitir potenciar la comunicación de los ODS y alcanzar así un mayor conocimiento y reconocimiento de estos y del sector.

Esta investigación también demuestra la oportunidad que tienen estas organizaciones de ponerse a la vanguardia. En un panorama de procesos lentos y donde algunas metas quedan muy alejadas, las organizaciones de la economía social tienen una oportunidad de comunicar su filosofía, sus valores y su alineación con los ODS. La comunicación de la Agenda 2030 y los ODS es el gran reto de los próximos 10 años.

\section{Bibliografía}

ALARCÓN CONDE, M.A. \& ÁLVAREZ RODRÍGUEZ, J.F. (2020): “El Balance Social y las relaciones entre los Objetivos de Desarrollo Sostenible y los Principios Cooperativos mediante un Análisis de Redes Sociales", CIRIEC-España, Revista de Economía Pública, Social y Cooperativa, 99, 57-87. DOI: 10.7203/CIRIEC-E.99.14322.

AFROBAROMETER (19 de noviembre, 2018): Taking stock: Survey findings track citizens' priorities, Sustainable Development Goals, and goverment performance in Africa. Recuperado de https://afrobarometer.org/press/taking-stock-survey-findings-track-citizens-priorities-sustainable-development-goals-and.

ALONSO, J.A. \& AYUSO, A. (coords.) (2017): Acercar los Objetivos de Desarrollo Sostenible a la ciudadanía: El compromiso de Europa con la Agenda 2030, CIDOB edicions, Barcelona. 
ARAUJO BATLLE, A., SERRANO ROBLES, E. \& JORDAN VALLVERDÚ, V. (2020): “La presencia de las cooperativas de Catalunya en Internet", CIRIEC-España, Revista de Economía Pública, Social y Cooperativa, 99, 37-56. DOI: 10.7203/CIRIEC-E.99.16902.

BERNAL JURADO, E., MOZAS MORAL, A., FERNÁNDEZ UCLÉS, D., MEDINA VIRUEL, M.J. \& PUENTES POYATOS, R. (2019): "Calidad de los sitios web en el sector agroalimentario ecológico y sus factores explicativos: el papel del cooperativismo", CIRIEC-España, Revista de Economía Pública, Social y Cooperativa, 95, 95-118. D0I: 10.7203/CIRIEC-E.95.13207.

BOTO-ÁLVAREZ, A. \& GARCÍA-FERNÁNDEZ, R. (2020): “Implementation of the 2030 Agenda Sustainable Development Goals in Spain", Sustainability, 12(6), 2546.

DOI: $10.3390 /$ su 12062546.

CARRICONDO, R., MOLERO, J., SÁNCHEZ, C. \& TOMÁS, M. (2019): Las ONG ante los Objetivos de Desarrollo Sostenible, Red Española del Pacto Mundial de Naciones Unidas, Madrid.

CASTIÑEIRA, Á. (dir.) (2019): La contribución de las empresas de las empresas españolas a los objetivos de desarrollo sostenible. Segundo informe, ESADE, Barcelona.

CASTIÑEIRA, Á. (dir.) (2020): La contribución de las empresas españolas a los objetivos de desarrollo sostenible, ESADE, Barcelona.

CENTRO DE INVESTIGACIONES SOCIOLÓGICAS [CIS] (2019): "Barómetro de febrero 2019. Distribuciones marginales", Estudios del CIS, 3240. https://cutt.ly/4dGyIR0.

CHAVES ÁVILA, R. \& MONZÓN CAMPOS, J.L. (2018): “La economía social ante los paradigmas económicos emergentes: innovación social, economía colaborativa, economía circular, responsabilidad social empresarial, economía del bien común, empresa social y economía solidaria", CIRIEC-España, Revista de Economía Pública, Social y Cooperativa, 93, 5-50.

DOI: 10.7203/CIRIEC-E.93.12901.

CONFEDERACIÓN EMPRESARIAL ESPAÑOLA DE ECONOMÍA SOCIAL [CEPES] (2018): Empresas relevantes de la economía social 2017-2018, CEPES, Madrid.

CONFEDERACIÓN EMPRESARIAL ESPAÑOLA DE ECONOMÍA SOCIAL [CEPES] (2019): La contribución de la economía social a los Objetivos de Desarrollo Sostenible. 4 INFORME sobre la experiencia de las empresas españolas de Economía Social en la Cooperación al Desarrollo 20172019, CEPES, Madrid.

COSTA, J. (1995): Comunicación corporativa y revolución de los servicios, Ediciones de las Ciencias Sociales, Madrid.

CRISTÓBAL FRANSI, E., MONTEGUT SALLA, Y. \& DARIES RAMON, N. (2017): “Cooperativismo 2.0: presencia en Internet y desarrollo del comercio electrónico en las cooperativas oleícolas de Cataluña", REVESCO. Revista de Estudios Cooperativos, 124, 47-73. DOI: 10.5209/REVE.54926. 
DAVANT, J.P. (2003): “Las organizaciones intersectoriales de la Economía Social: Francia, el CEGES”, En: VV.AA., La economía social y el tercer sector, 409-424. Escuela Libre Editorial, Madrid.

DNV GL (2020): Uniting Business in the Decade of Act, UN Global Compact, Nueva York.

ERRO SALA, J. (2002): Comunicación, desarrollo y ONGD, Hegoa, Bilbao.

EUROBARÓMETRO (№ 501) (2020): "Attitudes of European citizens towards the environment", Comisión Europea. https://cutt.ly/kfG1IJ0.

EUROBARÓMETRO (№ 494) (2019): “EU citizens and development cooperation”, Comisión Europea. https://cutt.ly/bfG10D5.

EUROBARÓMETRO (n- 441) (noviembre-diciembre 2015): “Cooperación y ayuda al desarrollo en la UE", Comisión Europea. https://cutt.ly/3fGMLX0.

GLESER, S.H. \& SCHNEIDER, S.H. (2018): Opinion monitor for development policy - Attitudes towards development cooperation and sustainable development, German Institute for Development Evaluation (DEval), Bonn. https://www.deval.org/en/evaluation-reports.html.

GOBIERNO DE ESPAÑA (2020): Informe de Progreso 2020. Reconstruir lo común, Gobierno de España. https://cutt.ly/ZfQZMc3.

GUAN, T., MENG, K., LIU, W. \& XUE, L. (2019): "Public attitudes toward Sustainable Development Goals: Evidence from five chinese cities", Sustainability, 11(20), 5793.

DOI: $10.3390 /$ su11205793.

HERRANZ DE LA CASA, J.M. (2007): "La comunicación en las organizaciones de la economía social", En: Salinas Ramos, F. \& Herranz De La Casa, J.M. (Coords.), Empresas de economía social: aspectos para su desarrollo, 61-96. Publicaciones Universidad Católica de Ávila, Ávila.

HERRANZ DE LA CASA, J.M., CANTERO DE JULIÁN, J.I. \& SIDORENKO BAUTISTA, P. (4-5 de octubre, 2018): "La gestión de la comunicación de las organizaciones representativas de la economía social en España. Análisis a través de su presencia en la red" [Comunicación en congreso], XVII Congreso Internacional de Investigadores en Economía Social y Cooperativa La Economía Social: transformaciones recientes, tendencias y retos de futuro, CIRIEC-España, Toledo.

HERRANZ DE LA CASA, J.M. \& GARCÍA, S. (2020): “Comprometidos con los ODS: cómo están comunicando las organizaciones los Objetivos de Desarrollo Sostenible", En: Herranz De La Casa, J.M. \& Gómez Ciriano, E.J. (Coords.), Los Objetivos de Desarrollo Sostenible desde una perspectiva de los Derechos Humanos, el Trabajo Social y la Comunicación, 25-54. Tirant lo Blanch, València.

JAVEED, A., YAR KHAN, M., REHMAN, M., KHURSHID, A. \& HASHMANI, M.A. (2020): “Tracking Sustainable Development Goals - A case study of Pakistan", Pre-Prints, 2020020201. D0I: $10.20944 /$ preprints202002.0201.v1 
JORGE VÁZQUEZ, J., CHIVITE CEBOLLA, M.P. \& SALINAS RAMOS, F. (2019): “La transformación digital en el sector cooperativo agroalimentario español: situación y perspectivas", CIRIEC-España, Revista de Economía Pública, Social y Cooperativa, 95, 39-70.

DOI: 10.7203/CIRIEC-E.95.13002.

MARTÍNEZ-CARRASCO PLEITE, F. \& EID, M. (2017): “El nivel de conocimiento y la reputación social de las empresas cooperativas. El caso de la Región de Murcia", CIRIEC-España, Revista de Economía Pública, Social y Cooperativa, 91, 5-29. D0I: 10.7203/CIRIEC-E.91.8865.

MENDOZA JIMÉNEZ, J., GUZMÁN PÉREZ, B., PÉREZ MONTEVERDE, M.V. \& ROMÁN CERVANTES, C.T. (2020): “The Contribution of the Fishermen's Guilds and the Agrarian Transformation Societies to the Sustainable Development Goals: The Case of the Canary Islands", Sustainability, 12(4), 5635. D0I: 10.3390/su12145635.

MOZAS MORAL, A. (2019): Contribución de las cooperativas agrarias al cumplimiento de los Objetivos de Desarrollo Sostenible. Especial referencia al sector oleícola, CIRIEC-España, València.

MOZAS MORAL, A. (coord.) (2019): "El cooperativismo y la economía social en la implementación de los ODS", Noticias de la Economía Pública, Social y Cooperativa (Noticias del CIDEC), Temas de Actualidad, 61, 36-75, Disponible en:

http://ciriec.es/wp-content/uploads/2020/05/Noticias_CIDEC61_web_tema.pdf.

NACIONES UNIDAS (2019): Informe de los Objetivos de Desarrollo Sostenible.

ORGANIZACIÓN INTERNACIONAL DEL TRABAJO [OIT] (2014): Las cooperativas y los Objetivos de Desarrollo Sostenible. Debate sobre el desarrollo después de 2015. Informe de política, OIT, Ginebra, https://www.ilo.org/empent/Publications/WCMS_307228/lang--es/index.htm.

PÉREZ BUENO, L.C. (dir.) (2019): Objetivos de Desarrollo Sostenible y promoción de los derechos de las personas con discapacidad, Ediciones Cinca, Madrid.

PLATAFORMA DEL TERCER SECTOR [PTS] (2020): Objetivos de desarrollo sostenible: un pacto para cambiar el mundo. Tercer Sector y Voluntariado, Plataforma del Voluntariado de España (PVE), Madrid. https://plataformavoluntariado.org/objetivos-de-desarrollo-sostenible-un-pacto-para-cambiar-el-mundo/.

RAMOS NAVARRO, M.T. (2019): “La contribución de Grupo Sorolla Educación a los ODS”, Noticias de la Economía Pública, Social y Cooperativa (Noticias del CIDEC), 61, 46-47.

https://dialnet.unirioja.es/servlet/articulo?codigo=7225536.

RED ESPAÑOLA DEL PACTO MUNDIAL DE NACIONES UNIDAS (2019): Las ONG ante los objetivos de desarrollo sostenible, Madrid.

RUIZ, E. \& GARCÍA, V. (2019): La hora del primer gran balance de la agenda 2030. Evaluando los avances de los ODS: una realidad fraccionada, Forética, Madrid. 
SACHS, J., SCHMIDT-TRAUB, G., KROLL, C., LAFORTUNE, G., FULLER, G. \& WOELM, F. (2020): The Sustainable Development Goals and COVID-19. Sustainable Development Report 2020, Cambridge University Press, Cambridge, https://cutt.ly/2fRACz7.

SALAMON, L. \& HADDOCK, M. (2015): SDGs and NPIs: private nonprofit institutions - The foot soldiers for the UN Sustainable Development Goals, Johns Hopkins Center for Civil Society Studies, Baltimore. http://ccss.jhu.edu/publications-findings/?did=451.

SÁNCHEZ, C., MOLERO, J., CARRIONDO, R. \& CASTILLO, E. (2020): Comunicando el Progreso 2019: Un análisis de los ODS y los Diez Principios en las empresas españolas, Red Española del Pacto Mundial de Naciones Unidas, Madrid.

SANZ DE LA TAJADA, L.A. (1994): Integración de la identidad y la imagen de la empresa, Editorial ESIC, Madrid.

SDG COMPASS (2015): The guide for business action on the SDGs. https://sdgcompass.org/download-guide/.

SILOS, J., RUIZ, P., CANALES, R., HERRERO, A. \& GRANDA, G. (2018): Informe Forética 2018 sobre la evolución de la RSE y Sostenibilidad. La recompensa del optimista, Forética, Madrid.

UNDP-MDG REPORT (2015): Informe de 2015 de los Objetivos de Desarrollo del Milenio, UNDP-MDG. https://cutt.ly/udRmMiq.

UNACC (2019): Quién es quién en las cooperativas de crédito, UNACC, Madrid.

WEIL, P. (1992): La comunicación global. Comunicación institucional y de gestión, Editorial Paidós, Barcelona. 
